\title{
«INTERPRETACIÓN SISTEMÁTICA»: ¿UN CONCEPTO REALMENTE ÚTIL? \\ Consideraciones acerca del sistema jurídico como factor de interpretación ${ }^{*}$
}

\section{Introducción}

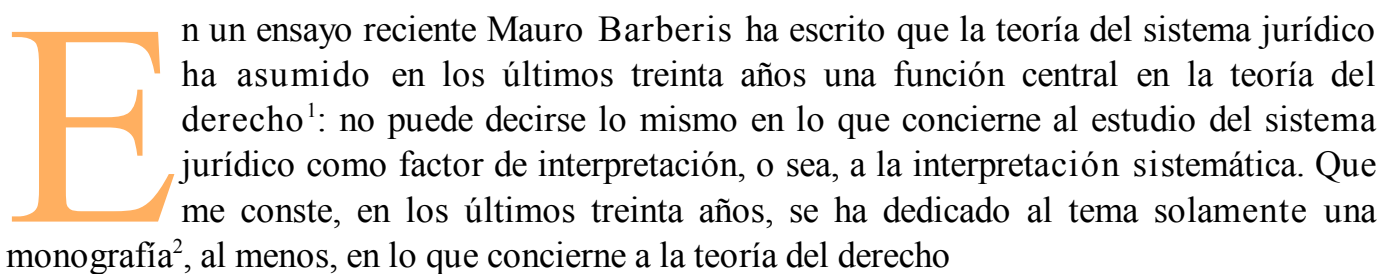

monografía ${ }^{2}$, al menos, en lo que concierne a la teoría del derecho

${ }^{*}$ Este artículo reproduce, con algunas modificaciones, el texto de la ponencia que presenté en el «Tercer seminario italiano-español de teoría analítica del derecho: Estructura y dinámica de los sistemas jurídicos», Sitges 15-18 octubre 1997. Agradezco a los organizadores por haberme permitido confrontar mis ideas en un contexto amistoso y estimulante.

${ }^{1}$ M. Barberis, Insiemi e sistemi. Un'obiezione ad Alchourron e Bulygin, ponencia presentada en el «Segundo seminario italiano-español de teoría analítica del derecho:"Estructura y dinámica de los sistemas jurídicos"», Madrid, Octubre, 1996. Trad. cast. Conjuntos y sistemas. Una objeción a Alchourrón y Bulygin, Doxa, Alicante, n. 20, 1997, pp. 26-52.

${ }^{2}$ Se trata del trabajo de G. Lazzaro, L’interpretazzione sistematica della legge, Turín, 1965; ya entonces el autor señalaba, al inicio del libro, la escasa atención prestada al tema, cfr. G. Lazzaro, L'interpretazione sistematica della legge, cit., p. 1: «Puede sorprender el hecho de que se haya prestado tan poca atención a la llamada interpretación sistemática en las numerosas monografias, de autores italianos y extranjeros, dedicadas a la interpretación en general o al método de la ciencia jurídica. Sin embargo, el recurso al sistema jurídico es una argumentación que se invoca frecuentemente para sostener tanto opiniones doctrinales como decisiones jurisprudenciales»; recientemente, se ha publicado un trabajo dedicado en apariencia a la interpretación sistemática, se trata del trabajo de V. Italia L'interpretazione sistematica delle norme e dei valori, Milán, 1993, al cual ha seguido la L'interpretazione sistematica delle regole giuridiche, Milán, 1997, obra reciente que es, en gran parte, un resumen de la anterior; en realidad, los textos citados no se refieren a los aspectos teóricos del tema que aquí nos interesa, sino que se ocupan de los condicionamientos interpretativos derivados de la relación jerárquica entre la constitución y la ley y entre la ley y el reglamento a la luz del derecho positivo italiano. Otra contribución dedicada al terna específico de la interpretación constitucional es el trabajo de P. Merola Chierchia, L'interpretazione sistematica della constituzione, Padua, 1978, en el que los elementos de la teoría general utilizada se han tomado prestados, en buena parte, de Lazzaro. Además, se debe hacer referencia al texto de M. Varejão, Intepretacão sistematica. Um exame da doutrina juridica italiana, Recife, 1994; la autora presta atención a la relación 
italiana, y, a pesar de que en el mismo periodo han aparecido muchos buenos trabajos sobre teoría de la interpretación, el espacio dedicado en éstos a la interpretación sistemática es escaso $^{3}$ y los análisis allí desarrollados no siempre resultan ser incisivos y precisos. Probablemente, esto se debe a la dificultad de racionalizar, de esquematizar el tema frente a la complejidad de la noción de sistema jurídico, o mejor, de las varias nociones de sistema, no necesariamente jurídico, a las cuales se puede recurrir desde la perspectiva de la interpretación ${ }^{4}$.

En las siguientes páginas mi intención es proporcionar un resumen de los desarrollos actuales de la interpretación sistemática en teoría del derecho, relativos a los argumentos interpretativos consolidados en la cultura jurídica continental, así como contribuir al análisis del concepto de interpretación sistemática, aclarando sus límites, en relación con otras técnicas interpretativas, con el fin de encontrar una respuesta a la provocativa pregunta del título de este artículo ${ }^{5}$. Una advertencia preliminar es obligatoria: se consideran aquí solamente los aspectos de la interpretación y no los de la integración jurídica, es decir, se consideran los métodos de atribución de significado a un texto normativo y no los métodos de producción de nuevas normas, a pesar de que éstos tengan también un alcance sistemático. Por lo tanto, no trataré la analogía en sus distintas formas, legis y juris ${ }^{6}$.

que existe entre interpretación y certeza del derecho, pero no analiza cada uno de los cánones hermenéuticos y su relación con las distintas nociones de sistema.

${ }^{3}$ Es una excepción R. Guastini, Le fonti del diritto e l'interpretazione, Milán, 1993; este libro contiene una articulada exposición de los argumentos sistemáticos y una clasificación de los mismos sobre la que volveremos frecuentemente en lo sucesivo, también en clave crítica.

${ }^{4}$ Como se verá más adelante, el recurso al sistema en la interpretación puede referirse, por ejemplo, al sistema jurídico en su conjunto o a un subsistema del sistema total en pocas palabras, la heterogeneidad de las nociones de sistema se refleja en las técnicas de interpretación haciendo heterogénea la noción de interpretación sistemática.

${ }^{5}$ Básicamente, lo que se pretende es llevar a cabo una operación de uso terapéutico del lenguaje (por usar el léxico tarelliano). Esta operación consiste en substituir un palabra ambigua, en nuestro caso «interpretación sistemática», por dos o más palabras nuevas, cada una de las cuales expresa sólo algunos de los posibles significados de la palabra original (acerca de este tema véase G. Tarello. Diritti, enunciati, usi, cit., pp. 403-406). Es decir, lo que se pretende es proporcionar una redefinición de la noción de interpretación sistemática (sobre el concepto de redefinición cfr. U. Scarpelli, Contributo alla semantica del linguaggio normativo, Milán, 1985, reimpresión editada por A. Pintore, p. 64 y ss.; así como R, Guastini, Distinguendo, Turín, 1996, p. 167: «[...] la redefinición consiste en precisar el significado de una palabra, eliminando, al menos en parte, la ambigüedad y la vaguedad que son propias del uso común».

${ }^{6}$ He recogido la distinción tradicional entre interpretación e integración, según la cual es conveniente hablar de integración del derecho y no de interpretación cuando se completa una disciplina en una materia dada o cuando se resuelven las antinomias (cfr. R. Guastini, voz Interpretazione, en Enc. Giur. Treccani, vol. XVIL Roma, 1989, p. 2; y G. Gavazzi, Elementi di teoria del diritto, Turín, 1984, p. 79). La operación por medio de la cual se previenen las antinomias 


\section{Los argumentos interpretativos sistemáticos}

No es oportuno aquí detenerse demasiado en el concepto de interpretación jurídica'; sin embargo, es necesario tratarlo brevemente con el fin de situar de un modo correcto y provechoso la interpretación sistemática y los argumentos interpretativos que se pueden reconducir a ella.

El término interpretación puede usarse tanto para indicar la actividad interpretativa como para indicar el resultado de esta actividad. El producto de la interpretación (actividad) es el significado del objeto interpretado ${ }^{8}$. La actividad interpretativa se puede clasificar de distintos modos, según cuáles sean los criterios utilizados, entre éstos revisten una importancia particular los criterios subjetivo y objetivo. En relación con el objeto es posible distinguir la interpretación de la ley, la interpretación del contrato, del acto administrativo, de la constitución, etc. ${ }^{9}$ Conforme al criterio subjetivo se puede

es, por el contrario, una actividad interpretativa y. en concreto, como se verá más adelante, es una interpretación sistemática. Otros se refieren a la distinción citada en términos de interpretación en sentido estricto e interpretación en sentido amplio. pero lo fundamental no cambia; en efecto, los argumentos interpretativos en sentido amplio son definidos del modo siguiente: «Se trata de principios y argumentos que justifican no la atribución de un significado a un texto jurídico, sino la producción de nuevas normas jurídicas a partir de las que aparecen expresadas en los textos jurídicos», así E. Diciotti. Norme, validitá, interpretazione giudiziale, ed. Provvisoria, Turín, 1996, p. 258.

${ }^{7}$ Para un resumen acerca de la interpretación jurídica, véase M. Jori - A. Pintore, Manuale di teoría generale del diritto, Turín, 1995, pp. 204-225.

${ }^{8}$ Esta distinción, ahora ya conocida, fue introducida por Giovanni Tarello en algunos trabajos recogidos en el volumen Diritti, enunciati, usi, Bolonia, 1974, fue recogida y desarrollada más adelante en L'interpretazione della legge, Milán, 1980, en particular, pp. 39 y ss., y está presente en la mayor parte de las obras de teoría de la interpretación de orientación analítica. Acerca de la distinción entre la disposición en cuanto objeto de interpretación y la norma en cuanto resultado, en cuanto producto, de la misma, véase, además de las aportaciones de Tarello. V. Crisafulli, voz «Disposizione (e norma)», en Enc. del dir. vol. XIII, Milán, 1964, p. 195 y ss., a pesar de que en Cristafulli los conceptos no se usen siempre de una manera clara e unívoca, sobre este punto cfr. R. Guastini, Dalle fonti alle norme, Turín, 1992, pp. 23-24.

\footnotetext{
${ }^{9}$ Es importante señalar, ya que es relevante para el examen del concepto de interpretación sistemática, que los tipos de interpretación jurídica mencionados pueden ser diferentes, y normalmente en la práctica lo son, según los subtipos de que se trate. Se puede distinguir la interpretación de la ley penal de la de las disposiciones normativas civiles, o la interpretación de las disposiciones administrativas de la interpretación de los actos administrativos que no son disposiciones. Se trata, como se verá mas adelante, de operaciones subsistemáticas; el intérprete no se refiere al sistema en su conjunto, sino a un particular sector del mismo en virtud de las características consideradas relevantes para los fines interpretativos. Acerca de la relevancia de este tipo de distinción cfr. E. Diciotti, Norme, validitá, interpretazione giudiziale, cit., p. 209. A veces, la distinción entre los subtipos se lleva a cabo porque en el sistema hay normas que regulan la interpretación de tipos particulares de textos. En el sistema italiano se pueden citar, a título de ejemplo, el art. 14 de las disposiciones preliminares al código civil, que se refieren exclusivamente a las leyes penales excepcionales, o las disposiciones sobre la interpretación del contrato arts. 1362-1371 del código civil italiano.
} 
distinguir entre la interpretación del juez, del abogado, del jurista. Esta distinción también es relevante dado que la diversidad de los sujetos intérpretes conlleva frecuentemente una diversidad de objetivos. La actividad interpretativa del juez está dirigida a la obtención de una regla de decisión de un caso concreto, la del jurista no tiene necesariamente tal objetivo $^{10}$. Las siguientes páginas se desarrollarán desde un punto de vista, en alguna medida, neutro en el plano subjetivo, es decir, se tratará la interpretación sistemática pasando, sin solución de continuidad, de la óptica del jurista a la del juez, y viceversa; aunque esté fuera de duda que la diversidad del sujeto intérprete, con la consecuente diversidad de objetivos, puede comportar una diferencia de opiniones respecto a la importancia, a la relevancia de cada uno de los argumentos interpretativos sistemáticos. Desde un punto de vista objetivo, el análisis versará sobre la ley, es decir, sobre las disposiciones normativas generales y abstractas.

Hasta aquí se han tratado algunas distinciones relativas a la interpretación (actividad, producto, etc.) sin definir, sin embargo, la interpretación jurídica ${ }^{11}$; interpretar quiere decir adscribir, atribuir un significado a un texto jurídico normativo, y esta atribución tiene lugar con base en argumentos interpretativos particulares. Precisamente, es sobre estos argumentos o métodos de interpretación sobre los que conviene que detengamos brevemente nuestra atención. Los argumentos interpretativos constituyen, como se ha dicho, el «medio» de atribución de significado al texto normativo y, por consiguiente,

\footnotetext{
${ }^{10}$ Más claro: el juez debe decidir un caso y, por lo tanto, tiene que obtener, a través de la interpretación, una regla para el mismo, llevando a cabo una elección entre las posibles interpretaciones del texto normativo. El jurista, el exponente de la doctrina, no tiene necesariamente que elegir entre las posibles interpretaciones del texto, pudiendo llegar a la conclusión de que varias soluciones del caso son adecuadas.

${ }^{11}$ Caracterizar la interpretación como adscripción, como atribución de significado a un texto normativo, implica aceptar una concepción escéptica de la interpretación según la cual pueden atribuirse diversos significados a un mismo texto. Como se ha señalado, esta concepción se opone al llamado formalismo interpretativo (sobre este tema y sobre el formalismo jurídico en general véase M. Jori, Il formalismo giuridico, Milán, 1980), según el cual, la interpretación consiste en comprender el único significado, el verdadero, el correcto, que tiene el texto. Jori aclara bien los caracteres del formalismo interpretativo, voz Formalismo giuridico, en Dig. disc. priv. sez. civ., vol. VII, Turín, 1992, p. 430: «El núcleo de estas concepciones [...] es, en cualquier caso, la afirmación de que es posible llegar a soluciones definitivas y exactas en la interpretación del derecho... Traducido en los términos apropiados de una teoría semiótica, esta afirmación no puede sino corresponderse a la tesis, semióticamente extravagante, de que cada enunciado jurídico posee, sólo o en conjunción con otros, un único significado propio o correcto». Las dos posiciones que he presentado de modo sumario en sus caracterizaciones extremas, son, en realidad, graduables; es decir, es posible sostener que la actividad interpretativa ni es arbitraria ni está totalmente constreñida, sino que el intérprete goza de alguna discrecionalidad interpretativa y que, por lo tanto, en cada una de las dos posiciones, la escéptica y la formalista, hay algo de verdad.
} 
representan la parte más relevante de la interpretación-actividad ${ }^{12}$. No existe acuerdo ${ }^{13}$ acerca de la naturaleza de estos argumentos, acerca de cuáles son estrictamente jurídicos y cuáles son sus características; las diferencias entre las distintas reconstrucciones dependen del hecho de que los argumentos interpretativos son predominantemente un producto cultura ${ }^{14}$; la atribución de significado a un documento normativo tiene lugar en un contexto histórico-cultural particular caracterizado por reglas y hábitos interpretativos. Estos hábitos interpretativos pueden referirse a los aspectos más variados: desde la jerarquía entre las normas, hasta las definiciones legislativas o las leyes interpretativas; respetar o contravenir estos hábitos constituye una razón para proponer o criticar un resultado interpretativo. En este mismo esquema se sitúan los argumentos interpretativos de los que nos

\footnotetext{
${ }^{12}$ Es conveniente distinguir entre la actividad interpretativa noética y la dianoética; los argumentos interpretativos están ligados a la segunda, es decir, a procedimientos discursivos y no intuitivos, cfr. L. Gianformaggio, Logica ed argomentazione nell 'interpretazione giuridica, en Studi Senesi, Milán, 1987, p. 465.

${ }^{13}$ Por ejemplo, escribe R. Alexy, A Theory of Legal Argumentation, Oxford, 1989, p. 3 y 234: «Even the number of these canons remains in dispute [...] The canons of interpretation have been the subject of wide ranging discussion ever since the time of Von Savign. Even today there is as yet no agreement as to their number, their precise formulation, their rank order, and their value». Un análisis detallado es el desarrollado por G. Tarello, L'interpretazione della legge, cit., p. 341 y ss., quien ha enumerado los siguientes argumentos: a contrario, analógico, a fortiori, de la plenitud del ordenamiento jurídico, de la coherencia del ordenamiento jurídico, psicológico, histórico, apagógico, teleológico, económico, natural, sistemático, de autoridad, de equidad y argumento a partir de principios generales; a continuación los ha clasificado en argumentos jurídicos y no estrictamente jurídicos, lógicos y no lógicos, precisando, en relación con los lógicos, que no se trata de argumentos lógicos en sentido estricto, es decir, formalmente restrictivos; argumentos completos e incompletos, conservadores y no conservadores. Para una reconstrucción reciente de las técnicas o métodos interpretativos véase R. Guastini, Le fonti del diritto e l'interpretazione, cit., pp. 358-408, ahora también en Distinguendo, cit., pp. 172-193; así como E. Diciotti, Norme, validitá, interpretazione giudiziale, cit., p. 250 y ss.

${ }^{14}$ He usado el adverbio «predominantemente» para no tomar una posición clara en relación con el papel que desempeña la retórica y la lógica en la interpretación jurídica, tema demasiado comprometido para ser abordado en pocas líneas. Sostengo, sin embargo, que es algo ya pacífico que la retórica ocupa un espacio relevante en la argumentación (cfr. G. Tarello, L'interpretazione della legge, cit., pp. 75-99, páginas en las que se discute ampliamente el papel de la lógica y de la retórica en el razonamiento interpretativo); otro problema es el de determinar de qué manera se puede establecer jerarquías o preferencias entre los distintos argumentos, cuál sea su fuerza. Sobre este tema véase la clara síntesis de G. Gavazzi, voz Topica giuridica, en Noviss. Dig. Italiano, vol. XIX, Turín, 1972, en particular, p. 415, y, como trabajo reciente, F. Cavalla, voz Tópica, en Enc. del dir, vol. XLIV, Milán, 1992, en especial p. 731 y ss. Acerca de la expresión «fuerza de los argumentos» cfr. Ch. Perelman y Olbrechts-Tyteca, Trattato dell'argomentazione. La nuova retorica, trad. it. de C. Schick, M. Mayer y E. Barassi, Turín, 1996, p. 483: «Para orientarse en la tarea interpretativa, el orador usa un concepto confuso pero que parece indispensable: el de fuerza de los argumentos».
} 
estamos ocupando ${ }^{15}$, respecto a los que se ha señalado que se trata de técnicas y métodos arraigados desde hace tanto tiempo en el uso jurídico que se pueden considerar específicos ${ }^{16}$. La caracterización de la interpretación jurídica como atribución de significado a un texto normativo, atribución que se lleva a cabo por medio de argumentos, de métodos interpretativos, permite distinguir entre dos tipos fundamentales de interpretación: la interpretación literal y la correctiva. Desde un punto de vista tradicional, interpretar literalmente significa atribuir a una disposición normativa el significado que le es propio, es decir, el significado más inmediato, ligado al uso común de la palabra; por el contrario, la interpretación correctiva se caracteriza por oposición a la literal, y es aquélla interpretación que corrige, que modifica el significado más inmediato de la disposición normativa. ${ }^{17}$ Esta distinción

15 Acerca del análisis de los argumentos interpretativos como instrumento de control del resultado interpretativo cfr. G. Tarello, L'interpretazione della legge, cit., p. 67: «El control, por parte de la sociedad, de las decisiones y de las propuestas acerca de la atribución de significado a los documentos que expresan las normas reguladoras de la vida social. se ejerce, entre otros modos, mediante el control sobre los procedimientos intelectuales que conducen a las decisiones y sobre los razonamientos que fundamentan la propuesta»; así como, R. Guastini (ed.), Problemi di teoria del diritto, Bolonia, 1980, pp. 214-215: «... la interpretación está sujeta a otro importante límite, digamos, fáctico. En cada ambiente cultural dado, los usos lingüísticos corrientes admiten una gama limitada -aunque vasta- de posibles significados de una expresión. Las atribuciones de significado que caen dentro de esta gama son toleradas; aquéllas que caen fuera de la misma son mal toleradas, hasta criticadas, o, simplemente, rechazadas. Por lo tanto, los intérpretes difícilmente pueden alejarse demasiado de los hábitos lingüísticos difundidos. Y entre éstos están incluidos naturalmente -si existen- las interpretaciones (jurídicas) ya propuestas y consolidadas de las expresiones objeto de interpretación». Sobre los límites de hecho de la interpretación cfr. G. Gavazzi, Elementi di teoria del diritto, cit., p. 77: «[...] sus límites están precisamente determinados por los usos lingüísticos que atribuyen ciertos significados a las palabras, que gobiernan el modo en el cuál las palabras se pueden combinar entre sí, y finalmente por la situación histórica en la cual el texto es producido e interpretado»; así como, F. Ost- M. van der Kerchove, Entre la lettre et l'esprit, Bruselas, 1989, pp. 32-34.

${ }^{16}$ Cfr. G. Tarello, Diritti, enunciati, usi, cit., p. 425; de todos modos, conviene señalar que ilustres teóricos del derecho han excluido que la interpretación jurídica sea claramente distinta de la interpretación en general, es decir, que tenga una especificidad propia; ésta es, por ejemplo, la opinión expresada por U. Scarpelli, L'interpretazione, premesse all teoria dell'interpretazione güiridica, en U. Scarpelli y V. Tomeo (eds.), Società, norme e valori. Studi in onore di R. Treves, Milán, 1984, especialmente, p. 162: «Yo me sitúo de hecho entre aquéllos que tienden a reconducir la interpretación jurídica al género de la interpretación...», no obstante, los términos de la polémica no son tan severos. De hecho, el mismo Scarpelli admite que la interpretación jurídica tiene peculiaridades. También Tarello utiliza frecuentemente para el análisis de la interpretación jurídica específica instrumentos teóricos de carácter general. Para un panorama general de los argumentos interpretativos nos remitimos a la nota 13 y a la bibliografía allí citada.

${ }^{17}$ A esta reconstrucción se refiere, entre otros, R. Guastini en Le fonti del diritto e l'interpretazione, cit., p. 359 y ss. El mismo autor reconduce a la interpretación literal los argumentos del lenguaje común y $a$ contrario, y a la interpretación correctiva todos los otros, incluso 
resulta útil para el análisis de los argumentos interpretativos sistemáticos, en cuanto que, como veremos, no todos éstos entran en relación con la interpretación literal del mismo modo, lo que nos permitirá realizar algunas distinciones, a mi modo de ver, interesantes.

Las someras consideraciones desarrolladas hasta aquí sobre el tema de la interpretación son suficientes para realizar una exposición de los argumentos interpretativos sistemáticos, tal y como éstos son utilizados en el discurso de los juristas ${ }^{18}$. Con la locución «interpretación sistemática» aquí nos referimos a una serie de técnicas y de argumentos interpretativos heterogéneos. Un modo típico de interpretar sistemáticamente consiste en hacer referencia a la situación de la disposición normativa ${ }^{19}$, lo que lleva a interpretar una cierta disposición de un determinado modo en relación con su situación.

Un segundo tipo de interpretación sistemática está constituido por la técnica de la disposición combinada, según la cual la norma está constituida por fragmentos de disposiciones normativas combinados entre sí.

En tercer lugar, se hace referencia a la interpretación sistemática cuando se utiliza el argumento de la constancia terminológica, según el cuál el legislador emplea un término siempre del mismo modo ${ }^{20}$.

los argumentos sistemáticos de los que nos ocuparemos después: es más, la interpretación sistemática viene a constituir una subespecie de la interpretación correctiva. La distinción entre interpretación literal y correctiva, aunque sea válida, debe adoptarse con las debidas precauciones en cuanto que, como se ha señalado, el concepto de significado literal es resbaladizo, el significado propio de las palabras es inasible (cfr. R. Guastini, Le fonfi del diritto e l'interpretazione, cit., p. 360), razón por la cuál también resulta difícil de determinar el concepto de interpretación correctiva. En relación con la vaguedad de las disposiciones normativas cfr. C. Luzzati, La vaghezza delle norme, Milán, 1990. No falta quien critica la distinción entre interpretación literal y correctiva no en el plano de las características del lenguaje (vaguedad, ambigüedad, etc.) sino en el plano de la propia teoría de la interpretación. Es decir, se sostiene que, incluso en los casos en los que se puede partir de un significado claro e inequívoco de una disposición, el intérprete debe ir más allá, atribuyendo a la disposición el significado más adecuado a los valores constitucionales y a los objetivos indicados en la constitución, por lo que la operación hermenéutica se caracteriza por una unidad substancial (para este planteamiento cfr. P. Perlingieri, L'interpretazione della legge come sistematica ed assiologica, il broccardo in claris non fit interpretatio, el rtiolo dell'art. 12 disp. Prel. c.c. e la nuova scuola dell'esegesi, en ID, Scuole, tendenze e metodi, Nápoles, 1989, pp. 273-299).

${ }^{18}$ Como ya he dicho para esta exposición me baso en la reconstrucción proporcionada por R. Guastini, Le fonti del diritto d l'interpretazione, cit., p. 377-383, ya que se trata, según me consta, del análisis más reciente.

${ }^{19} \mathrm{El}$ argumento de la sedes materiae, junto a la consideración del contenido de otras normas constituye, para G. Lazzaro, la interpretación sistemática por excelencia, L'interpretazione sistematica delle legge, cit., en particular, pp. 113-127.

${ }^{20}$ Por ejemplo, sosteniendo que el término posesión empleado en varias disposiciones penales en materia de delitos contra el patrimonio se deba entender en el sentido que dispone el art. 1140 del Código Civil, que proporciona una definición de posesión. 
También el argumento de la inconstancia terminológica constituye una expresión típica de la interpretación sistemática. El uso de este argumento está en función de la construcción de los subsistemas, ya que se sostiene que no es que el legislador no haya usado el mismo término siempre del mismo modo, sino que el término tiene diversos significados en razón de los diversos contextos en los que se utiliza ${ }^{21}$.

También se interpreta de un modo sistemático cuando se recurre a las construcciones dogmáticas para atribuir significado a una disposición normativa ${ }^{22}$.

Hay también una subespecie de interpretación sistemática, llamada adaptativa, según la cual a una disposición normativa debe atribuírsele el significado que se adapte mejor, que se adecue, al significado atribuido a otras disposiciones jerárquicamente subordinadas.

El argumento de la coherencia es una de las expresiones típicas de la interpretación sistemática y se utiliza con el fin de hacer coherente el sistema jurídico, es decir, para prevenir, para evitar las antinomiaS ${ }^{23}$ y las contradicciones normativas en general.

Como se puede observar, a partir de este breve análisis, la noción de interpretación sistemática se utiliza con relación a argumentos interpretativos heterogéneos, precisamente, porque detrás de cada técnica interpretativa

${ }^{21}$ Retomando el ejemplo de la nota anterior: el término posesión se entiende en el ámbito penal en un sentido diverso al que establece el código civil, en virtud de la especificidad del «sistema» penal.

${ }^{22}$ El ejemplo más evidente está constituido, en la doctrina civil italiana, por el concepto de negocio jurídico. Se trata de la llamada construcción jurídica, es decir, de aquella operación que consiste en subsumir un hecho o un acto dado en un concepto dogmático, producto de la dogmática. Acerca de la construcción jurídica cfr. G. Lazzaro, Storia e teoria della costruzione giuridica, Turín, 1965. El método de la construcción jurídica ha sido el primer método considerado como interpretación sistemática desde el punto de vista de la interpretación, sobre este punto véase la reconstrucción de K. Larenz, Storia del metodo della scienza giuridica, trad. it., Milán, 1966, p. 3 y ss.

${ }^{23}$ Es una opinión difundida, tanto entre los que aplican el derecho como entre los teóricos del derecho, que los sistemas jurídicos a pesar de que no sean coherentes deben tender a la coherencia. Sobre los conceptos de plenitud y coherencia de los sistemas normativos consúltese el análisis de C. E. Alchourrón - E. Bulygin, Normative Systems, Wien-New York, 1971. Acerca del concepto de antinomia, véase G. Gavazzi, Delle antinomie, Turín, 1959, y A. Ross, Diritto e giustizia, trad. it. de G. Gavazzi, Turín, 1965, p. 112: «Existe una incompatibilidad entre dos normas jurídicas cuando los efectos jurídicos incompatibles se refieren a los mismos hechos típicos». Sin embargo, en el texto se ha hecho referencia a las contradicciones normativas en términos generales y no necesariamente a aquellas particulares contradicciones que son las antinomias; se ha usado el término coherencia en relación con las paranomias, es decir, para referirnos no a una «mera contradicción lógica entre las normas» sino «... a algo más, es decir, a la relación que existe entre entidades que no solamente no son incompatibles entre sí, sino que también están ligadas por alguna conexión». En este sentido, véase A. Constanzo, Condizioni di incoerenza, Milán, 1992, p. 23 (de la introducción). Además, no faltan las reconstrucciones 
hay una noción particular de sistema ${ }^{24}$. En las siguientes páginas, pretendo analizar cada uno de los argumentos citados, con el fin de evaluar en cuáles de ellos la noción de sistema desempeña un papel relevante para los fines interpretativos y en cuáles, por el contrario, la referencia al sistema desempeña un papel secundario, para algunos casi despreciable, no esencial y subordinado al desempeñado por otros argumentos interpretativos. Más claro:

más o menos recientes que adoptan una noción de antinomia más amplia que la atribuible a Ross, mencionada más arriba, que no comprende exclusivamente las contradicciones lógicas entre normas, según el uso que he hecho en el texto del término antinomia, sobre este punto cfr. P. Chiassoni, Le sentenze civili. Istruzioni per l'uso, en prensa, Giuffré, Milán: así como K. Engisch, Introduzione al pensiero giuridico, trad. it., Milán, 1970, p. 255 y ss., en particular, p. 257, quien sugiere usar la noción de antinomia para referirse a cualquier perturbación de la unidad del ordenamiento jurídico. No es indispensable, y tampoco sería posible hacerlo, tratar aquí el concepto de congruencia, y sus relaciones con la coherencia, concepto que desde hace tiempo ocupa el debate teórico anglosajón. Aquí nos limitamos a remitirnos para un resumen de este debate y para los referencias relativas al mismo en la abundante literatura que existe a A. Schiavello, Su «congruenza» $e$ "diritto»: un'analisi comparata di modelli diversi, Ragion pratica, Génova, 1998, en prensa.

${ }^{24}$ No es causalidad que en teoría del derecho los argumentos que se reconducen a la interpretación sistemática sean diversos según los autores. R. Alexy, A Theory of Legal Argumentation, cit. p. 234 y ss. considera argumentos sistemáticos: «[...] both to the position of a norm in a legal text, and to the logical or teleological relation of a norm to other norms, goals and principles». Otros autores se acercan al tema con extrema cautela, afirmando que la interpretación sistemática «[...] includes inter alia the following arguments 1) the use of a statutory provision for interpreting another such provision; 2) interpretation influenced by the systematic of the statute; 3) interpretation influenced by another type of conceptual analysis; 4) interpretation influenced by other legal-dogmatic theories», A. Peczenick, On Law and Reason, Dordrecht, 1989, p. 384. Aarnio (cfr. The Rational as Reasonable, Dordrecht, 1987, pp. 126-128) considera como sistemáticos los argumentos de coherencia y los de las construcciones dogmáticas. Otros (cfr. Tarello, L'interpretazione della legge, cit., pp. 375-378) reconducen a la interpretación sistemática los argumentos de la constancia terminológica, dogmático y topográfico, precisando que se trata de argumentos que tienen en común poco más que el nombre, es decir, el ser de algún modo sistemáticos. Además, se debe añadir que, en la literatura italiana relativa al tema de la interpretación jurídica de tradición no analítica, se alude frecuentemente de manera enfática aunque poco útil al elemento sistemático de la interpretación con el fin de atribuir significado a un texto normativo (cfr. E. Betti, L'interpretazione della legge e degli atti giuridici, Milán, 1949, pp. 284-289, que se expresa en términos de «momento sistemático» de la interpretación sin especificar con claridad el modo en que tal «momento» se refleja en la interpretación del texto normativo). A título de ejemplo quisiera subrayar algunos datos relativos a la práctica judicial italiana. En una investigación empírica que estoy llevando a cabo sobre la base de un conjunto de voces (interpretación sistemática, interpretación lógico sistemática, exigencias 
sistemáticas, sistema de derecho), inherente a las decisiones tanto de los tribunales inferiores como de los superiores, ha resultado que el recurso expreso a la interpretación sistemática, a las voces mencionadas arriba, desde 1979 hasta hoy, es verdaderamente escaso. Son solamente ciento dieciséis las decisiones en las cuáles se recurre a las voces utilizadas para la investigación y en muchas de ellas se usa la interpretación sistemática como sinónimo de la analogía o como mero soporte de la interpretación literal. Para un cuadro comparado consúltese N. MacCormick- R. Summers, Interpreting Statutes, Darmouth, 1991, en especial, pp. 26-43 y 513-518. 
intento analizar individualmente las técnicas interpretativas llamadas sistemáticas, para poner en evidencia los diversos conceptos de sistema a los cuales se refieren y la preponderancia, en algunas de ellas, de otros factores (literal, teleológico) determinantes para la atribución o la elección entre los posibles significados del texto normativo.

\section{Interpretación y sistema}

Se ha visto, a partir de las contribuciones más recientes, cuáles son los argumentos interpretativos sistemáticos, y no es difícil advertir la variedad de las nociones de sistema a las que se hace referencia en el empleo de estos argumentos. Sin embargo, antes de analizar individualmente las distintas técnicas interpretativas con el objetivo de realizar la operación de terapia lingüística de la que se ha hablado ${ }^{25}$, conviene detenerse en la noción de sistema jurídico, para valorar la relevancia que tiene esta noción en relación con la interpretación; de todos modos, se puede ya avanzar que la abundante literatura que existe sobre el sistema jurídico no es de gran ayuda para el estudio de la interpretación sistemática, y pido disculpas al lector por el tono desenvuelto y excesivamente sintético de los pasajes de la siguiente exposición.

Se entiende por sistema jurídico un conjunto no vacío de elementos entre los cuáles subsisten diferentes relaciones ${ }^{26}$. Las relaciones entre los elementos pueden venir dadas por el orden en el cual el teórico exponga el resultado de su propia investigación (sistema extrínseco), por un cuerpo de doctrina de uno o más juristas, o por «[...] cualquier cosa que no sea relativa al modo de exponer un conjunto de conocimientos, sino el objeto mismo de una investigación teórica [...] esta segunda noción puede llamarse sistema intrínseco y puede especificarse para indicar: una totalidad deductiva de discurso cualquier totalidad o todo organizado [...] de organismo y de unidad finalística $\rangle^{27}$ (sistema intrínseco). Se ha señalado correctamente que, desde este último punto de vista, el sistema «[...] peut etre également conçue, soit dans une perspective "formalle" comme repondant à des exigenses de clartè, de non redondance, de cohèrence et de complétude, soit dans une perspective plus "materialle" et axiologique comme rèpondant à

\footnotetext{
${ }^{25}$ Véase nota 5.

${ }^{26}$ Éste es el núcleo de la definición que constituye el punto de partida del análisis desarrollado por M. G. Losano, Sistema e struttura nel diritto, vol. 1, Turín, 1968. La literatura italiana y extranjera en el tema del sistema jurídico es vastísima, nos remitimos a los textos ya citados y a la bibliografía indicada en los mismos.

${ }^{27}$ G. Lazzaro, voz Sistema giuridico, en Noviss. Dig. Italiano, vol. XIX, Turín, 1970, p. 459.
} 
un souci d'harmonie dans le contenue des règles appartenant à un même ensemble» ${ }^{28}$.

Esta idea de sistema como unidad finalista fue aplicada al derecho por los exponentes de la escuela histórica; en este sentido, las palabras de von Savigny son emblemáticas: «El orden de una obra sistemática está naturalmente determinado por esa íntima conexión que se debe reflejar en ella. A menudo se quiere pensar sólo en esto cuando se habla de tratamiento sistemático, [...] las relaciones jurídicas de la rica y vívida realidad forman un todo orgánico, que nosotros, sin embargo, estamos constreñidos a descomponer en sus partes para comprenderlas sucesivamente y para poderlas comunicar a otros ${ }^{29}$. Por consiguiente, para von Savigny, el derecho es un sistema puesto que el mismo no es considerado simplemente como un conjunto de normas diversas, sino como un organismo que se refleja en sus normas ${ }^{30}$. No hay duda de que la idea de sistema como un todo unitario y coherente ha tenido una influencia determinante en el pensamiento de los teóricos posteriores, a pesar de haberse introducido frecuentemente en contextos histórico-jurídicos distintos de aquéllos en los que von Savigny había madurado su pensamiento. Asimismo, la influencia ha sido relevante también en el campo de la interpretación al ser el jurista alemán el defensor más autorizado de la interpretación sistemática ${ }^{31}$. En su obra, como se ha destacado ${ }^{32}$, se diferencian claramente los aspectos teleológicos de la interpretación de los estrictamente sistemáticos y es siguiendo esta línea de pensamiento, releyéndola a la luz del análisis de los rasgos característicos o deseables de los sistemas jurídicos, que es posible reexaminar, aclarar, el concepto de interpretación sistemática.

\footnotetext{
${ }^{28}$ F. Ost- M. Kerchove, Entre la lettre et l'esprit, cit., p. 57. 21.

${ }^{29}$ F. C. von Savigny, Sistema del diritto romano attuale, trad. it. de V. Sciajola, Turín, 1886,p.

${ }^{30}$ Conviene aclarar que en von Savigny la interpretación se caracteriza como una actividad
} intelectual, y el sistema es una obra predominantemente del jurista dogmático, a quien corresponde, en ausencia de leyes o de códices, que no son realmente necesarios para el pensador alemán, la labor de sistematizar y convertir en orgánico el derecho. En relación con la mayor parte de los ordenamientos continentales contemporáneos, basados en la codificación, el concepto de interpretación sistemática de von Savigny ya no está vigente, puesto que los códigos son la base del sistema incluso en lo que concierne a la interpretación y los protagonistas, si se puede decir así, no son ya los dogmáticos sino los juristas.

31 Para ser más exactos, von Savigny individualiza cuatro elementos de la interpretación: gramatical, lógico, histórico y, precisamente, sistemático, cfr. F. C. von Savigny, Sistema del diritto romano attuale, cit., pp. 212 y ss.

${ }^{32}$ D. Farias, Interpretazione e logica, Milán, 1990, pp. 232-248, donde se demuestra que la interpretación lógica de von Savigny corresponde a la hoy denominada interpretación teleológico-objetiva, y que la interpretación sistemática está caracterizada por la necesidad de armonizar la norma en el sistema, en un todo orgánico. 
Por otra parte, es una opinión extendida entre los teóricos del derecho y entre los juristas que el ordenamiento jurídico o sistema ${ }^{33}$ es un conjunto de normas válidas; al asumir que el derecho es un ordenamiento o sistema se quiere decir que el derecho «[...] es algo ordenado, sistemático, no caótico y, precisamente por ello, unitario, tendencialmente coherente, y (quizá incluso) tendencialmente completo ${ }^{34}$. La caracterización del sistema como conjunto de normas tendencialmente coherente da lugar a problemas particulares desde la perspectiva de la interpretación. En realidad, los sistemas jurídicos se presentan como un conjunto de disposiciones, de textos normativos, siendo las normas, como se ha visto, el producto, el resultado de la interpretación. Además, los textos normativos son susceptibles de interpretaciones múltiples y variables, razón por la cual «[...] la representación de los ordenamientos jurídicos como conjuntos de normas (en sentido propio) oscurece el hecho de que los actos que habitualmente consideramos como normativos -como ejemplo paradigmático tenemos los actos legislativos producen, en rigor, no ya normas, hechas y terminadas, sino más bien textos normativos, cada uno de los cuáles es susceptible: por un lado, (a) de interpretaciones sincrónicamente múltiples y, por otro, (b) de interpretaciones diacrónicamente cambiantes» ${ }^{35}$. Por lo tanto, el ordenamiento jurídico no es algo dado, sino que sobre él incide profundamente la interpretación, dado que las normas son el resultado de la misma. Por ello, me parece que se puede

${ }^{33}$ Usos los términos «ordenamiento» y «sistema» jurídico como sinónimos, a pesar de que la sinonimia no sea pacífica; sobre este tema cfr. G. Tarello, Prospetto per la voce «Ordinamento giuridico» de una enciclopedia, en Politica del diritto, 1975, pp. 73-102; así como para un útil resumen de la evolución de los conceptos de sistema y ordenamiento jurídico a partir del siglo XIX, M. Barberis, Insiemi e sistemi, cit., en particular. pp. 2-9.

${ }^{34}$ R. Guastini, voz Ordinamiento giuridico, en Dig. disc. pubbl., vol. X, Turín, 1995, p. 4 (del extracto). La coherencia entendida como no contradicción entre normas se considera como una condición deseable de los sistemas jurídicos, a la cual deben tender los intérpretes, M. van de Kerchove - E Ost, Le système juridique entre ordre et desordre, Bruselas, 1988, p. 83: «Quant à la cohérence tout d'abord, on ne peut nier l'importance d'une telle propriete, que ce soit au niveau des idéax généraux qui polarisent en permanance l'activité et la pensée juridiques, ott que ce soit au niveau de la définition plus spécifique de la notion même de système juridique». En este sentido, véase también la obra clásica de N. Bobbio, Teoria dell'ordinamento giuridico, Turín, 1960, p. 80.

${ }^{35}$ R. Guastini, voz Ordinamento giuridico, cit., p. 6 (del extracto). M. van de Kerchove -F. Ost afirman que el sistema jurídico es una entidad mutable, cambiante cuyo orden se forma pasando por un desorden (y viceversa) determinado precisamente por las inevitables antinomias, por la pluralidad de intereses, de conflictos, que caracterizan necesariamente a los sistemas jurídicos, Le système juridique entre ordre et desordre, cit., pp. 233-240. Del tema de la relación entre sistema e interpretación se ha ocupado también A. Aarnio, The Rational as Reasonable, cit., p. 128: «In other words, the result of the interpretation depends on the systemic whole, and the systemic whole depends on the content that the norm being interpreted receives. The system is not given in advance (once for all) but is partially formulated on the basis of interpretation», opinión que comparto. 
afirmar que, en el plano de la evolución histórica y de las características que se considera que un sistema, que un ordenamiento jurídico, debe alcanzar, el concepto de interpretación sistemática se puede comprender mejor si se refiere sólo, o preferentemente, a los argumentos interpretativos que conllevan una atribución de significado, o una elección entre más significados posibles, por medio de la cual se hace coherente el sistema, evitando, previniendo, las posibles contradicciones entre normas ${ }^{36}$.

\section{Subsistemas, interpretación teleológica y coherencia del sistema jurídico}

A la luz de lo que se ha expuesto hasta aquí es fácil apercibirse de la ambigüedad de la noción de interpretación sistemática, bien en relación con el registro de los hábitos interpretativos extendidos en el ambiente jurídico, o bien, en lo que concierne a las reconstrucciones realizadas por reconocidos teóricos del derecho. Por lo tanto, conviene intentar reducir la ambigüedad de la noción objeto de examen, analizando cada uno de los argumentos interpretativos sistemáticos. Me ocuparé de los distintos métodos sistemáticos de atribución de significado distinguiendo entre la interpretación sistemática en sentido fuerte y en sentido débil, según que los distintos argumentos se refieran o no al sistema jurídico entendido en su conjunto, a su «coherentización» con el objetivo de evitar las antinomias, o a partes y visiones extrínsecas del sistema jurídico, y no con el objetivo de la coherencia, en el sentido aclarado en las páginas anteriores de unidad formal y material de este sistema ${ }^{37}$, siendo otros argumentos interpretativos, el teleológico en particular, los que asumen una relevancia primaria en la atribución de significado, con la esperanza de llevar a cabo una operación clasificatoria que haga útil, es decir, menos ambiguo, el concepto de interpretación sistemática $^{38}$.

${ }^{36}$ En este sentido también S. Soler, La interpretación de la ley, Barcelona, 1962, pp. 110-112. R. Alexy sostiene que es sistemática en sentido estricto la interpretación relativa a la exclusión, a la prevención de los conflictos entre normas, A Theory of Legal Argumentation, cit., p. 240. Este autor prefiere definir los otros argumentos de naturaleza sistemática como sistemático-teleológicos, poniendo en evidencia el predominio de este último aspecto.

${ }^{37}$ Cfr. nota 23.

${ }^{38}$ Es necesario aclarar las relaciones entre el «objetivo de la coherencia» y los argumentos interpretativos. Estos últimos pueden ser divididos en dos tipos fundamentales: de primer y de segundo grado. Los argumentos de primer grado son aquéllos por medio de los cuales se atribuye significado a un texto normativo; son de segundo grado los métodos, las técnicas interpretativas utilizadas para elegir, o para apoyar, una determinada atribución de significado respecto a otras atribuciones efectuadas con los argumentos de primer grado. Por lo tanto, la coherencia, la cohesión entre las normas, se puede obtener a través de cualquier técnica interpretativa (teleológica, literal, a contrario, etc.), sin embargo, lo que caracteriza la interpretación sistemática es, según mi modo de ver, el punto de vista del intérprete, que se fija el objetivo de la coherencia y de la armonía entre las normas como decisivo en la elección entre las posibles atribuciones de significado. Por consiguiente, como trataré de explicar a continuación, los argumentos de segundo grado son reconducibles a la interpretación sistemática propiamente dicha. 
Argumento de las sedes materiae o topográfico. Conforme a este argumento, debe atribuirse significado a las formulaciones legislativas a partir de los indicios que se pueden obtener de la situación de la disposición en el texto normativo ${ }^{39}$. En realidad, este argumento es de naturaleza textual y no propiamente sistemática ${ }^{40}$, en cuanto que la idea de sistema aquí no desempeña un papel determinante en la atribución de significado, sino que, en buena medida, está ligada al contexto literal-textual, es decir, al significado que las disposiciones asumen en el lenguaje ordinario o técnico-jurídico y en su conexión sintáctica, así como a la indagación de la intención del legislador ${ }^{41}$, sin que haya aquí una relación estrecha entre la atribución de significado al texto normativo y el sistema jurídico entendido como un sistema tendencialmente coherente. Además, la referencia topográfica no recurre al sistema jurídico sino al sistema de la ley o al subsistema en el cual se sitúa la disposición. Este argumento es sistemático en sentido débil.

Argumento de la constancia terminológica. Conforme a este argumento, debe atribuirse el mismo significado a los mismos términos recurrentes en los textos normativos. Esto significa que, al atribuir significado a un término contenido en una formulación normativa, se debe tener en cuenta el significado que esta expresión asume en otras disposiciones normativas. El

${ }^{39}$ Esta técnica interpretativa se fundamenta en el presupuesto de la racionalidad del legislador conforme al cual este dispone de manera ordenada y no casual. Este modo de pensar se puede conectar con la idea misma de sistema entendida en sentido extrínseco, como método de disposición. Acerca del principio de racionalidad del legislador véase E Ost, L'interpretation judiciaire et le postulat de rationalité du legislateur, en M. van de Kerchove- F. Ost, Jalons pour une thèorie du droit, Bruselas, 1987, pp. 355-419.

${ }^{40}$ Se puede decir con E. Diciotti, Norme, validità e interpretazione giudiziale, cit., p. 245, que se trata de un método textual, conforme al cual se considera a las disposiciones normativas no sólo en su significado literal sino también en relación ron el ámbito textual en el que éstas se sitúan (así también, G. Gavazzi, Elementi di teoria del diritto, cit., p. 78: «La interpretación sistemática puede considerarse textual si las disposiciones pertenecen al mismo texto»). Por lo que la dicotomía inicial interpretación declarativa e interpretación correctiva puede substituirse por la dicotomía interpretación textual y correctiva. A la interpretación textual son reconducibles todos los argumentos conectados con el ámbito textual de la disposición, a la interpretación correctiva todos los otros, entre los cuales se pueden incluir también los argumentos interpretativos sistemáticos entendidos en sentido fuerte.

${ }^{41}$ Como se ha señalado, el argumento teleológico puede ser reconstruido en sentido subjetivo y objetivo. En el primer caso, se hace referencia a la intención del legislador histórico, utilizando como fuente reconstructiva de la misma los trabajos preparatorios; en el segundo caso, se hace referencia a la voluntad de la ley, desconectada del legislador histórico, determinada de modo objetivo, devaluando así el papel de los trabajos preparatorios. Sobre este tema véase el reciente trabajo de A. Moscarini, Ratio legis e valutazioni di ragionevolezza della legge, Turín, 1996, así como A. Peczenick, On Law and Reason, cit., pp. 346-355 y 404-418. 
intérprete puede mantener la constancia terminológica sólo en el ámbito de la misma ley o de un determinado ámbito disciplinar (penal, civil...); por consiguiente, se trata de una interpretación sistemática en sentido débil, puesto que el intérprete está motivado por consideraciones de carácter predominantemente teleológico. En efecto, atribuir el mismo significado al mismo término en el interior de la misma ley o del mismo ámbito disciplinar implica sostener que existen razones, objetivos, finalidades ligadas a la ley o un ámbito disciplinar particular, que determinan la necesidad de un uso constante de los mismos términos ${ }^{42}$. Sostener que un término deba entenderse del mismo modo en cualquier texto normativo en que se repita, independientemente del sector disciplinar en el que se sitúe la disposición normativa en que éste se encuentre, supone realizar una interpretación sistemática también en sentido débil, ya que se considera el sistema, el ordenamiento jurídico, en una visión unitaria, total, no fraccionada, pero sólo debido a un presupuesto de razonabilidad lingüística del legislador o a razones teleológicas y no con la finalidad de hacerlo coherente.

Argumento de la inconstancia terminológica. Al utilizar este argumento el intérprete atribuye a un término un significado distinto del que éste tiene en otras normas, sosteniendo que conviene hacerlo en cuanto que exigencias particulares así lo requieren. A mi modo de ver, esta técnica interpretativa tiene una naturaleza teleológica y no estrictamente sistemática. En efecto, el intérprete argumenta sobre la base de consideraciones subsistemáticas, es decir, en relación con los objetivos, con las razones, relativas al sector disciplinar al que pertenece la disposición normativa. En el ámbito penal, interpretar la noción de posesión de modo diferente al que dispone el art. 1140 del código civil, significa realizar una operación teleológica, ya que se defiende que, en virtud del interés protegido en las disposiciones penales en las que aparece el término posesión, éste no puede entenderse del mismo modo que en el ámbito civil ${ }^{43}$. Por lo tanto, este argumento es sistemático

${ }^{42} \mathrm{~K}$. Engisch. Introduzione al pensiero giuridico, cit., pp. 120-121, considera imposible escindir la interpretación sistemática de la teleológica: «Puesto que la relación sistemática de cada norma con el ordenamiento jurídico en su conjunto es en gran parte de naturaleza teleológica, dado que las normas generalmente tienen la finalidad de realizar, junto a otras normas, determinados objetivos [...] es muy difícil separar la interpretación sistemática de la teleológica». Está fuera de duda que las dos formas de interpretación, teleológica y sistemática, están relacionadas, el problema no es poner en evidencia este hecho sino identificar criterios de distinción satisfactorios que permitan que las dos nociones ocupen espacios de recíproca autonomía y que no terminen, por el contrario, en una confusa sinonimia.

${ }^{43}$ Para ser más claros: en los delitos contra el patrimonio (hurto, apropiación indebida, etc., regulados en los artículos 624-649 del código penal italiano), el término posesión frecuentemente recurrente debe entenderse como dominio autónomo sobre la cosa y no como ejercicio sobre la cosa de un poder correspondiente al derecho de propiedad o a otro derecho real, tal y como está establecido en el artículo 1140 del código civil, puesto que el interés protegido en las normas penales en cuestión (determinado en vía subjetiva u objetiva), exige esta interpretación y no la que se deriva del argumento de la constancia terminológica. 
en sentido débil al ser un instrumento de la interpretación teleológica objetiva o subjetiva que sea.

Argumento de la disposición combinada. Este argumento es, a mi modo de ver, particularmente complejo y de difícil clasificación. En efecto, muchos de los ejemplos citados para aclarar el contenido de esta técnica interpretativa no son indicativos. Es recurrente el ejemplo de la determinación del significado de una disposición sobre la base de otras disposiciones a las que ésta reenvía de manera expresa o implícita ${ }^{44}$. Entendido de este modo, el argumento no es sino un supuesto de determinación de una norma por medio de otras disposiciones y nada más, careciendo, por lo tanto, de una autonomía conceptual particular. Realmente, no me parece que tenga una relación particular con la interpretación sistemática como no sea en el sentido débil y obvio de que para atribuir significado a un texto normativo es necesario tener en cuenta las conexiones que existen entre este texto y otras disposiciones normativas ${ }^{45}$.

Argumento de la coherencia, de la interpretación adaptativa y de las construcciones dogmáticas. Éstas son las tres técnicas interpretativas sistemáticas en sentido fuerte, ya que se refieren al sistema entendido en un sentido unitario, como un todo que debe tender a la coherencia, a la prevención de las contradicciones normativas.

Apelando a la coherencia se descarta toda posible atribución de significado a una disposición que determine una divergencia, un conflicto con el contenido de cualquier otra disposición ya interpretada. No es estrictamente necesario que entre las normas exista una relación jerárquica.

Una aplicación particular del argumento de la coherencia viene dado por la interpretación adaptativa, en la que entra en juego el problema de las jerarquías normativas y axiológicas. Se refiere a la adecuación de significado de una disposición al significado (ya establecido) de otras disposiciones de rango superior (como ejemplo paradigmático encontramos la interpretación constitucionalmente orientada de las disposiciones legales); pero también se hace referencia a la interpretación adaptativa cuando se adecua el significado de la disposición normativa a un principio general del derecho, establecido

${ }^{44}$ Para algunos ejemplos cfr. R. Guastini, Le fonti del diritto e l'interpretazione, cit., p. 378.

${ }^{45}$ En el mismo sentido del texto cfr. M. Jori- A. Pintore, Manuale di teoria generale del diritto, cit., p. 213: «Por lo tanto, interpretar el derecho siempre quiere decir componer y relacionar los significados relevantes de muchísimas disposiciones jurídicas... el caso más simple y evidente de esta labor de composición es la investigación de lo que la práctica jurídica llama la "disposición combinada" [combinado disposto]». 
con antelación. En el primer caso, la interpretación está condicionada por una jerarquía formal, en el segundo, por una jerarquía que se puede definir, de un modo aproximativo, como axiológica ${ }^{46}$.

También el uso de construcciones dogmáticas con fines interpretativos constituye una forma de interpretación sistemática en sentido fuerte, puesto que el uso de una construcción jurídica, de un concepto general elaborado por el intérprete, sirve para armonizar, para hacer coherentes las disposiciones objeto de interpretación ${ }^{47}$. El uso de la construcción dogmática constituye una interpretación más que sistemática, «sistematizante», puesto que el intérprete construye un sistema de relaciones normativas compatible con la construcción dogmática utilizada ${ }^{48}$.

\section{Algunas conclusiones}

No sé si haya logrado clarificar el concepto de interpretación sistemática. En resumen se puede decir que: 1) es conveniente dividir el concepto de interpretación sistemática en dos subclases: interpretación sistemática en sentido fuerte y en sentido débil. La distinción se fundamenta en el hecho de que a la primera son reconducibles aquellos argumentos que permiten hacer coherente el sistema, que permiten la prevención de las antinomias, de los conflictos entre las normas, asumiendo este aspecto una función fundamental en la atribución, y/o en la preferencia, de un significado, en la corrección en sentido restrictivo o extensivo del significado del texto normativo. A la segunda subespecie (interpretación sistemática en sentido débil), son reconducibles los otros argumentos interpretativos comúnmente considerados sistemáticos (inconstancia terminológica, etc.), ya que, en estos casos, no se prefiere el fin de la coherencia entre las normas, y la atribución

${ }^{46}$ En este sentido, R. Guastini, Le fonti del diritto e l'interpretazione, cit., pp. 381-383, en particular, p. 383: «Claramente, las diversas formas de interpretación adaptativa tienen el objetivo (y el efecto) de evitar el surgimiento de antinomias entre las normas de distinto grado jerárquico, o entre las normas particulares y los principios generales, según los casos. Por esta razón, la interpretación adaptativa siempre puede ser argumentada -y habitualmente lo es- recurriendo al dogma de la coherencia del derecho». Como trabajo reciente sobre las diversas formas de jerarquía normativa véase: R. Guastini, Gerarchie normative, en Materiali per una storia della cultura giuridica, 2/97, Bolonia, 1997, pp. 463-487, en particular, pp. 470-471, donde se enumeran cuatro tipos de jerarquías entre normas y se aclaran los conceptos de jerarquía formal y axiológica. Acerca del concepto de "principio del derecho" nos remitimos a la vastísima literatura existente sobre la materia, buena parte de la cuál puede encontrarse en R. Guastini, voz Principi di diritto, en Dig. disc. priv. sez. civ., vol. XIV, Turín, 1996, pp. 34 1-354.

${ }^{47}$ En efecto, ésta ha sido la operación realizada por la Pandectística alemana en el siglo XIX al elaborar conceptos generales que permitieran una sistematización orgánica; un ejemplo que ya he citado es el concepto de negocio jurídico.

${ }^{48}$ Debo esta última observación al Prof. Ernesto Garzón Valdés. 
de significado se lleva a cabo en función de criterios textuales o teleológicos. Además, en estos casos, el «sistema» al que se hace referencia está dado por el contexto textual de la disposición o por el ámbito disciplinar al que ésta pertenezca. 2) El concepto de coherencia al que se ha hecho referencia en el texto abarca no sólo las contradicciones lógicas entre las normas sino también la cohesión de las mismas.

(Trad. de Amalia Amaya) 